inappropriate admission (Flannigan et al, 1994). If so, junior psychiatrists appear to be good judges of appropriateness of admission, since most admissions considered inappropriate lasted only a few days. However, despite considering admission to be inappropriate and having the option of other services, junior psychiatrists repeatedly admitted patients. Perhaps the ease of admission was attractive when alternative resources may be difficult and time consuming to access, or junior psychiatrists may consider particular patient groups threatening or unpredictable. Although statistical analysis did not reach significance, there is a suggestion that suicidal ideation was more common in cases than controls. It is possible that the expression of suicidal ideation acts as a form of talisman in the patient gaining admission. This along with previous suggestions warrants further study.

Perhaps the key point to emphasise is the importance of auditing the admission process. Flannigan et al (1994) suggested that clinicians need to identify those patient groups that benefit from admission with consultants offering supervision to more junior gatekeepers. The findings of this study support this view.

\section{Acknowledgement}

I thank Dr David Owens, Senior Lecturer in Psychiatry, University of Leeds for help in analysing the results.

\section{References}

Beck, T. J., Croudace, J., Singh, S., et al (1997) The Nottingham acute bed study: alternatives to acute psychiatric care. British Journal of Psychiatry, 170. 247-252.
Creed, F., Mbaya, P., Lancashire, S., et al (1997) Cost effectiveness of day and inpatient psychiatric treatment: results of a randomised controlled trial. British Medical Journal, 314, 1381-1385.

Flanigan, C. B., Glover, G. R., Wing, J. K., et al (1994) Inner London collaborative audit of admission in two health districts. III: Reasons for acute admission to psychiatric wards. British Journal of Psychiatry. 165. 750-759.

Gofrman. E. (1961) Asylums. New York: Doubleday.

GOLDBERG, D. \& HUXLEY, P. (1980) Mental Ilness in the Community: the Pathway to Psychiatric Care. London: Tavistock.

Hicks, N. R. (1994) Some observations on attempts to measure appropriateness of care. British Medical Journal, 309, 730-733.

HouLT, J. (1986) Community care of the acutely mentally ill. British Journal of Psychiatry, 149, 137-144.

Kent, S. \& Yellowlees, P. (1994) Psychiatric and social reasons for frequent rehospitalization. Hospital and Community Psychiatry, 45, 347-350.

Meyerson, A. T., Moss, J. Z., Belville, R., et al (1979) Influence of experience on major clinical decisions. Archives of General Psychiatry, 36, 423-427.

SCAMBLER, G. (1991) Sociology as Applied to Medicine. London: Baillière Tindall.

STEIN, L. I. \& TEST, M. A. (1980) Alternative model to mental hospital treatment. I. Conceptual model, treatment program. and clinical evaluation. Archives of General Psychiatry, 37, 392-397.

Strumwasser, I., PARANuPE, N. V., Udow, M., et al (1991) Appropriateness of psychiatric and substance abuse hospitalization: implications for payment and utilization management. Medical Care, 29 (suppl.). S77-S90.

WORLD HEALTH ORganization (1992) The Tenth Revision of the International Classification of Diseases and Related Health Problems (ICD-10). Geneva: WHO.

Peter Y. Elwood, Consultant Psychiatrist, Peter Hodgkinson Centre, County Hospital, Greetwell Road, Lincoln LN2 5UA

\title{
Solo obstetric liaison service
}

\author{
Sue Smith
}

Aims and method To set up a liaison psychiatry service for an obstetric department and review how much of the work involved in such a service can be undertaken by a senior registrar in two special interest sessions. Results It was clear there was a demand for a service for women with psychiatric problems associated with childbirth. Referrals from colleagues in general psychiatry meant that the available time was soon used up. This did therefore not allow time to develop efficient systems for referral and management or to then see the extra referrals this would have produced. The limited and temporary service was well recelved by 
other professionals and by patients. The number of referrals received fell far short of the expected morbidity.

Clinical implications in an area with this number of births and its consequent level of psychiatric morbidity, it would not seem possible, within two special interest sessions, to develop a more formalised or comprehensive system.

A woman has the greatest lifetime risk of being admitted to a psychiatric unit within the first 90 days after childbirth (Kendall et al, 1987). Puerperal psychosis is relatively rare occurring after $0.05 \%$ of deliveries. Non-psychotic postnatal depression is the most common serious complication of the postnatal period, following $10-15 \%$ of deliveries (Pitt, 1968; Kumar \& Robson, 1984); $1.7 \%$ of all post-partum deliveries are referred to psychiatric services (Kendell et al, 1987; Oates, 1994). A number of risk factors have been identified as important in psychosis and non-psychotic depression (O'Hara et al, 1984; Warner et al, 1996) offering potential for prevention.

A College working party in 1992 recommended that women requiring psychiatric services following childbirth should be treated by a consultant psychiatrist who has a special interest in their condition, supported by a multi-disciplinary team (McClelland, 1992).

A comprehensive perinatal psychiatric service leads the organisation of appropriate care for all women with serious mental illness following childbirth. This needs a multi-disciplinary approach from staff with specialist experience and skills. Close liaison between maternity services, general adult mental health services, primary care and social services is essential with establishment of protocols for prediction and early detection. Education and training should also be a priority.

The purpose of this article is to describe what aspects of perinatal work were achieved during one year by a senior registrar in her two special interest sessions.

At the time of writing, the Department of Psychiatry in Southampton had 75 in-patient beds for general adult psychiatry and 20 beds for rehabilitation. It covers a catchment area of 525000 , including areas of inner-city deprivation.

The Southampton area has an annual delivery rate of approximately 5500 . This could be expected to generate over 500 cases of major depression annually, 5-10 cases of puerperal psychosis, and 85-100 referrals (Oates, 1994).

At the start of this work, there were no local dedicated beds for admitting mothers with their babies, and no local consultant psychiatrist with a special interest in perinatal psychiatry. Mothers requiring admission with their babies had gone to the mother and baby unit in
Basingstoke, some $\mathbf{3 0}$ miles away or to a somewhat nearer private establishment.

Prior to April 1996, a senior registrar had established an out-patient clinic at the maternity hospital to see referrals from both the obstetric and gynaecology services. From 1 April 1996 I took up post as a third year senior registrar working in the rehabilitation psychiatry service. I intended to use my two special interest sessions to set up a formalised referral system for the maternity services to refer pregnant or postnatally depressed mothers who were ill or 'at risk'. I had some previous experience of working in this area. I did not intend to take on gynaecology referrals.

Before I was able to set up clear protocols for referral and circulate them to the maternity services, my interest quickly became known among my psychiatric colleagues. They eagerly referred me anybody who was, had recently been or was thinking of becoming pregnant! I soon accumulated a case-load of women who I saw in different settings for varying lengths of time. This left no time for development of procedures such as a formal referral system or screening protocol.

Supervision was available from the consultant responsible for liaison psychiatry, and also from regular monthly sessions with a consultant psychotherapist.

The following figures are an accumulation of the work I did over that year, April 1996 to March 1997. The total number referred to me was 26 , and I saw 24 . The mean age was 27 years (range 17-36).

\section{Mode of referral}

Ten of the 24 referrals came via the maternity services. The first three were handed over from the previous senior registrar clinic, the remaining seven were first seen via the liaison rota (a rota of junior and senior psychiatric staff responsible for assessing overdoses and liaison referrals on any particular day). These were then directed to me from colleagues involved in that rota, or on call on a particular day. Most of the other referrals came directly from psychiatric colleagues. Two were from a nurse on the neonatal intensive care unit and three from general practitioners who came to know of my interest.

\section{Reason for referral and initial diagnosis}

I was interested in the concept of primary prevention and how intervention in pregnancy with women who were well but 'at risk' may reduce the risk of postnatal mental illness. However, only four women were well at the time of referral and concerned about risk of recur- 
Table 1. Reason for referral

\begin{tabular}{lc}
\hline & $\boldsymbol{n}$ \\
\hline $\begin{array}{l}\text { Antenatal } \\
\text { Previous postnatal moderate depressive } \\
\text { episode, currently well }\end{array}$ & 3 \\
Mild depressive disorder & 2 \\
Moderate depressive disorder & 2 \\
Eating disorder & 3 \\
Bipolar disorder, currently well & 1 \\
Other & 3 \\
Total & 14 \\
Postnatal & \\
Moderate depressive episode & 1 \\
Severe maternity 'blues' & 2 \\
Puerperal psychosis & 1 \\
Other & 3 \\
Total & 7 \\
\hline
\end{tabular}

Table 2. Outcome at end of year

\begin{tabular}{lr}
\hline & $n$ \\
\hline Discharged to general practitioner & 11 \\
Transferred to general psychiatrist & 2 \\
Transferred to psychotherapy service & 1 \\
Transferred to new perinatal service & 4 \\
Declined or did not attend further follow up & 6 \\
Total & 24 \\
\hline
\end{tabular}

rence of a previous postnatal depression. The remaining 10 referred antenatally were symptomatic, seven with depressive symptoms, three with an eating disorder. Of those referred postnatally, two were seen soon after delivery with severe maternity 'blues'.

There was a wide range of diagnoses, including one borderline personality disorder and one case of post-traumatic stress disorder. The three referrals not included in the tables were a mild depressive disorder secondary to infertility, a bereavement reaction following a stillbirth and preconception advice to a woman who had suffered a puerperal psychosis with her first baby (see Table 1).

\section{Urgency of referral}

An urgent response was requested on six occasions, usually when women were distressed just after childbirth. The majority of requests were routine, but in order to take advantage of the opportunity for preventative work pointed out by Kendell (1997) it would seem appropriate to see an 'at risk' woman as early on in pregnancy as possible (Table 2).

\section{Place of initial assessment}

Despite concerns expressed by maternity services about women feeling stigmatised at having to attend a psychiatric hospital, over half were happy to be seen here. Most of the others were seen at home, one was first seen at the maternity hospital, and one at the general hospital following an overdose.

\section{Treatment offered}

This varied from support and monitoring for emergence of symptoms to managing a case of puerperal psychosis in the community. Where they existed, the local community mental health teams were always informed of my involvement, but in most cases were not directly involved.

Well women seen during pregnancy to discuss risk of recurrence were seen once or twice before the birth, followed up once at around two or three weeks and then again two months later providing depression did not develop before that. In some of these cases, prophylactic antidepressants were started immediately after delivery. Where the woman was seen depended on her preference, time and space availability.

\section{Communication}

In perinatal liaison work, communication with the other professionals involved is extremely important. Most women with postnatal depression are treated in primary care, and health visitors are particularly well placed to detect and help manage psychological morbidity at this time. My initial method of communication was by phone calls which were appreciated as an efficient means of facilitating shared care. To follow-up the telephone conversations, 86 letters were written in total, mainly to the general practitioners with copies to other involved professionals.

\section{Outcome at end of year}

No quantitative measures of outcome were used. Numbers were too small and the sample too heterogenous to be able to draw meaningful conclusions about the mental health outcomes of the women seen. Table 2 shows what happened to the women when I left Southampton at the end of the year.

\section{Other activities}

I met once with health visitors to discuss their plans to set up protocols for detecting and managing postnatal depression. I provided minor input to the neonatal intensive care unit, attending a ward round and seeing mothers there that staff were concerned about and doing a teaching session on postnatal depression for the staff. I was involved in other educational 
activities, for example giving lectures to primary care groups.

\section{Conclusion}

My findings mirrored a previous study whose aim was to pick up 'at risk' women and work preventatively. They found the majority of referrals to a new obstetric liaison service were for women with psychiatric symptoms during pregnancy (Appleby et al, 1989). The wide spectrum of psychiatric disorders referred was noted in another study (Dunsis \& Smith, 1996), and this was replicated in my experience.

It was clear from my experience that there was a demand for a service for women with psychiatric problems associated with childbirth. Because of the pressure on the general psychiatric service, colleagues were keen to pass on their cases so that my available time was soon used up. This did therefore not allow me time to develop efficient systems for referral and management or to then see the extra referrals this would have produced.

Towards the end of the year, the health authority developed a contract for specialist perinatal services covering the area. This service will develop more formalised provisions and include an in-patient facility as well as outreach community-based services.

The limited and temporary service I was able to offer was well received by other professionals and by patients. I always discussed cases with referrers who felt my input was valuable, but it was not possible to more formally monitor outcomes. It provided me with good experience of the variety of psychiatric conditions presenting during and after pregnancy. The number of referrals I received during this period fell far short of the expected morbidity. However, to have further advertised my service would have led to an unmanageable workload for the time available. It is evident that in an area with this number of births and its consequent level of psychiatric morbidity, it would not be possible, within two special interest sessions, to develop a more formalised or comprehensive system.

\section{References}

APPLEBY, L., Fox, H., SHAw, M., et al (1989) The psychlatrist in the obstetric unit. British Journal of Psychiatry. 154. 510-515.

Dunsis, A. \& SMITH, G. C. (1996) Consultation-liaison psychiatry in an obstetric service. Australia and New Zealand Journal of Psychiatry, 30. 63-73.

KENDELL, R. E. (1997) How psychiatrists can contribute to the public health. Advances in Psychiatric Treatment, 3. 188-196.

-. Chalmers. L. \& Platz, C. (1987) The epidemiology of puerperal psychosis. British Journal of Psychiatry. 150. 662-673.

KUMAR, R. \& RoBson, K. M. (1984) A prospective study of emotional disorders in childbearing women. British Journal of Psychiatry, 144. 35-47.

MCCLELLAND. R. (1992) Working group on mental health information systems. Psychiatric Bulletin, 16. 522-523.

OATES. M. R. (1994) Postnatal mental illness: 'Organisation and function of a psychiatric service'. In Aspects of Perinatal Psychiatry: Use and Misuse of the EPDS. London: Gaskell.

O'HARA. M. W., Neunaber. D. J. \& ZeKosKI. E. M. (1984) A prospective study of postnatal depression: prevalence. course and predictive factors. Journal of Abnormal Psychology, 93, 158-171.

PrTT, B. (1968) Atypical depression following childbirth. British Joumal of Psychiatry. 114. 1325-1335.

WARNER, R. APPLEBY, L., WhITTON, A., et al (1996) Demographic and obstetric risk factors for postnatal psychiatric morbidity. British Joumal of Psychiatry. 168, 607-611.

Sue Smith, Consultant in General Adult and Perinatal Psychiatry, Sully Hospital, Hayes Road, Sully, Vale of Glamorgan CF64 5YA

\title{
Bridging the psychotherapy divide
}

\author{
Mary T. Monaghan and Stirling Moorey
}

Aims and method All consultant psychotherapists should be sufficiently familiar with the three major branches of psychotherapy, to appropriately match therapy with their assessment of the patients' needs. This requires that specialist registrars training in psychotherapy acquire skills in psychodynamic/ interpersonal, cognitive-behavioural and systemsbased therapies.

Results While acknowledging the benefit for the trainee of being familiar with more than one model of 